\title{
Users' View on Context-Sensitive Car Advertisements
}

\author{
Florian Alt, Christoph Evers, Albrecht Schmidt \\ Pervasive Computing Group \\ University of Duisburg-Essen \\ Schuetzenbahn 70, 45119 Essen, Germany \\ \{florian.alt, albrecht.schmidt\}@uni-due.de \\ christoph.evers@stud.uni-due.de
}

\begin{abstract}
Cars are ubiquitous and offer large and often highly visible surfaces that can be used as advertising space. Until now, advertising in this domain has focused on commercial vehicles, and advertisements have been painted on and were therefore static, with the exception of car-mounted displays that offer dynamic content. With new display technologies, we expect static displays or uniformly-painted surfaces (e.g. onto car doors or the sides of vans and trucks) to be replaced with embedded dynamic displays. We also see an opportunity for advertisements to be placed on non-commercial cars: results of our online survey with 187 drivers show that more than half of them have an interest in displaying advertising on their cars under two conditions: (1) they will receive financial compensation, and (2) there will be a means for them to influence the type of advertisements shown. Based on these findings, as well as further interviews with car owners and a car fleet manager, we discuss the requirements for a context-aware advertising platform, including a context-advertising editor and contextual content distribution system. We describe an implementation of the system that includes components for car owners to describe their preferences and for advertisers to contextualize their ad content and distribution mechanism.
\end{abstract}

\section{Introduction}

Cars are ubiquitous in today's societies and, given their size and shape, offer potential space for placing highly-visible advertising. Vehicles are used and seen by people, and can therefore lead to many points of visual contacts between people and cars. For example, a pedestrian seeing cars driving by, a driver or passenger driving behind another car and looking at its rear, and a person walking by a row of parked cars at a parking lot and glancing at each of them. Until now, very few privately-owned cars have become advertising spaces and commercial vehicles have mostly static advertisements that promote the services and products of the company that bought them. Since the early 90 s, vehicles used in public transport have been utilized as advertising space. One of the first examples is the Pepsi advertising campaign that started in 1993, where the Pepsi logo was painted on urban buses in the Seattle area. Since then, advertising on vehicles has remained mostly static, in the sense that the ads do not adapt to their immediate situation or context. The first approaches to offer dynamic advertising content involved mounting electronic displays on cars where the content 
might be location dependent, e.g. taxis in the Boston area ${ }^{1}$. With advances in display technologies, we anticipate that there will be new ways for attaching or embedding electronic displays into car surfaces, such as car doors or the sides of vans and trucks. This will create new opportunities for advertising. We argue that context will play a major role for efficiency and acceptance of advertising displays.

We explore the potential of cars as dynamic and contextual advertising space in this paper. In section 2, we present the results of an online survey with 187 drivers summarizing their attitudes towards car-based advertising. Based on these findings, we describe in section 3 the requirements, concept and design space for a contextual advertising system for vehicles. In section 4 , we describe an implementation of the central components and a platform of a dynamic advertising system prototype to show its feasibility. We then discuss related work in this domain and further opportunities and implications of our work in the conclusion.

The contribution of this paper is twofold:

- A detailed analysis of users expectations on context advertising on vehicles that reveals an interest in providing advertising space on privately-owned cars in return for an incentive (e.g. money) if there is an appropriate way for users to influence which advertisements are shown.

- A discussion of the design space and requirements for a platform that provides a means for creating and deploying contextual advertising to be shown on cars and a proof-of-concept implementation based on these requirements.

\section{Car owners' expectations on contextual car advertising}

To understand users' expectations, motivations, and constraints for providing their car as a platform for advertising, we conducted an online survey and follow-up interviews with car owners and a car fleet manager. We hope that by looking broadly at potential target groups-from private car owners to large companies with company car fleets-, we can provide a more comprehensive picture of the requirements.

\subsection{Privately-owned cars: Online survey}

In our survey, we were especially interested in 1) when users want to make their car available for advertising, 2) product categories users would be willing to show advertisements for, 3) the acceptance of different technological solutions, and 4) possible rewards for showing advertisements on private vehicles.

The survey was completed by 187 persons, 130 males and 57 females. The average age of the participants was 27.4 years (25.2 among males vs. 23.3 among females). The majority of participants were driving compact-sized vehicles (128) and mediumsized vehicles (41). Recruitment for the survey was done via email.

\footnotetext{
${ }^{1}$ Taxis with roof mounted signs that change their content according to the GPS position, see http://www.clearchanneltaximedia.com/products/taxi-tops-digital-smart.asp for details.
} 
First, we asked for the preferred reward schema. We suggested different types of rewards, including monthly allowance, discount on car purchase, benefits from third parties (coupons for cinema/concerts, etc.), and coupons for free fuel. On a 5-point Likert scale ( $1=$ not interesting at all, 5=definitely interesting), 155 of 187 participants were interested (gave a rating of 3 or higher) in a monthly allowance, 139 in a discount upon purchasing the car and 137 in coupons for free fuel. Only 50 subjects were interested in receiving coupons from third parties as a reward.

Then, we asked when users would like to provide their cars as advertising space. Users were asked to choose from several options: at any time, while driving, while I am not close to my car, and while I cannot see my car. We found that 105 subjects $(56.1 \%)$ did not care when advertisements are displayed on their car, while 37 subjects $(19.8 \%)$ preferred not to be close to their car while it was showing ads 24 subjects $(12.8 \%)$ did not want to see their car while it was advertising.

Next, we evaluated the effect of the advertised products on the attitude of car owners towards advertisements. We provided users with a selection of different categories, including music, clothes, fast food, beverages, tobacco, firearms, politics, culture, eroticism, and sport events. We found that users preferred not to show advertisements on their cars for controversial categories. These included tobacco (124 subjects, $66.1 \%$ ), eroticism (144 subjects, $77.0 \%$ ), firearms (151 subjects, $80.8 \%$ ), and politics (116 subjects, 62.3\%). On the other hand, they strongly preferred showing advertisements on culture (173 subjects, 92.5\%), music (167 subjects, 89.3\%), sport events (161 subjects, $86.1 \%$ ), and clothes (151 subjects, $80.7 \%$ ).

Additionally, we looked into the acceptance of different technical solutions. We suggested four options to the users: painting, easy-to-detach foil, fixed labels, and roof-mounted equipment. Using a 5-point Likert scale (1=inacceptable, 5= acceptable), we found that the most popular solution among the participants was easy-todetach foil (92.0\% with a rating of 3 or better), followed by fixed labels $(55.62 \%)$. Rather unpopular were painting (43.85\%) and roof-mounted equipment (19.78\%).

Finally, we wanted to know which parts of the car participants preferred to have the advertisements appear on. Participants used a 5-point Likert scale to evaluate the following locations: rear/trunk, rear windshield, rear side windows, sides/doors, roof, front lid. The result shows that most participants favor the sides/doors $(83.9 \%$ with a rating of 3 or better), followed by the vehicle's rear/trunk $(78.6 \%)$, roof $(77.01 \%)$, rear side windows $(67.4 \%)$, rear windshield $(60.9 \%)$ and front lid $(60.43 \%)$.

The survey revealed the following findings with regard to privately-owned cars:

(1) Incentives are essential for convincing users to provide their cars as advertising space. Preferred rewards for placing advertisements on cars are monthly allowance, discounts upon purchasing a car, and coupons for free fuel.

(2) More than half of the participants would not care if they were close to their car while ads were being shown. However, one third preferred not to have the ads shown when they were close by or could see the ads themselves.

(3) Users want to stay in control of the products advertised, especially those falling under controversial categories, such as eroticism, firearms, and tobacco.

(4) For technical solutions, easy-to-detach foil was rated by far the most preferred option while roof-mounted equipment was rather unpopular.

(5) The side doors, trunk and roof are among the favorite areas on the car for placing advertisements. 


\subsection{Privately-owned cars: Qualitative interviews}

In order to gain further understanding of the car owners' views on car advertising, we conducted 4 follow-up interviews with people who participated in the online survey. The subjects were two students (each 21 years old), a contractor (50), and a real estate administrator (45). First, we asked them about concrete incentives and values. All subjects would be interested in a monthly allowance $(80 €-120 €$ monthly), two of them would also like to receive coupons for free fuel $(80 €-100 €$ monthly). Next, we asked the subjects about how to select the products they would be willing to advertise on their cars. Two favored a category list, where they could select groups of products, and the other two favored positive lists for a more fine-grained selection. However, all of them wanted to be able to update the list at any time. Finally, we asked about privacy concerns regarding contextual and location information that might be transmitted about their car, e.g. GPS data. Three of the subjects were not concerned at all, adding that they were participating in payback programs anyway. However, all four subjects stated that their GPS data should not be provided to the advertisers.

\subsection{Company-owned cars: Interview with a car fleet manager}

To complement the view of privately-owned cars with that of large companies that own cars for business use, we conducted a telephone interview with a fleet manager of a pharmaceutical company, who is responsible for a fleet of approximately 600 cars with a replacement rate of 200 cars/year. During the interview, we discovered issues and concerns regarding the hypothetical introduction of car advertisements in the fleet. One of the manager's primary concerns was the additional administrative workload. This additional workload would be partly due to the need to equip cars with the required technical gear and partly due to the necessity to define products that should not appear as ads on the fleet's vehicles. The manager also felt that a monthly allowance of about $10 \%$ of the monthly leasing rate of the cars would be an attractive incentive. A final and interesting finding was with regard to the acceptance among the employees using the cars. Since cars are a part of an employee's compensation, as well as a status symbol, the manager felt that it would be essential (especially among sales personnel) to allow the employees to have a strong influence on the decision of whether or not to allow advertising on their cars.

\section{Contextualized Advertisements on Cars}

In this section, we outline the design space for a system for context-aware car advertisements.

Means for expressing preferences. One central requirement is that car owners have the power and means to specify what advertisements are shown on their car. It is also essential that the system is easy to use. Potential solutions include giving the user a 
list of products and brands that she or he can accept or deny (not individually but as a whole). Asking the user to provide preferences for each product may lead to a lengthy and tedious process and asking for each product category may be too general to accurately specify their preferences. Therefore, we suggest creating a preferences editor based on a combination of product groups and brands. This approach scales well to large numbers of advertisements and appears to satisfy the users' concerns.

Simple technical solution. The technical solution for advertising must be very simple to apply and remove. A major change to the appearance of the car (e.g. display system on the roof) is also not welcomed. The required effort by the car owner for administrating, configuring, and maintaining such a display surface must be minimal. One potential technical solution is a self-contained display unit consisting of a magnetic foil (to make it easily attachable to the car door) with a bendable display (e.g. eInk display) on top and built-in communication and context-sensing. This unit would potentially be powered by energy harvesting.

Defining context. In the case of contextualized advertisements on vehicles, the advertising message would be broadcasted to all people who focus directly or indirectly on the car. Thus, the audience is not limited to a specific user but rather to a group defined by context. We identified the following crucial context information:

- Spatial: Location is an important factor when dealing with context-aware ads. Using knowledge of the geographic location of the display, ads can be targeted at the audience expected in that region. It may also be possible to estimate the potential view context (e.g. distance of the observer to the car).

- Demographic: From spatial information, demographical data can be derived, such as information on the people living, working, or staying in that area.

- Temporal: For the advertiser, knowing when ads are shown is important. The temporal context can either be date- and/or time-based or even further abstracted (e.g. during rush hour, lunch break, after work).

- Weather: For several product groups (e.g. cloth, drinks, food), the current weather conditions, temperatures and forecasts may be relevant to deciding which ads to display.

- History: Past information on displayed advertisements can facilitate the decision-making process when other context-information is unavailable.

Gathering context. There are two steps to gathering the context: (1) collecting objective data from the context using sensors embedded in the display, and (2) complementing the sensor data with external information. For the first step, location data via a GPS sensor is an obvious option but additional sensors that provide information about proximity (e.g. WLAN or Bluetooth scan) or weather conditions are also possible. Other types of context information (e.g. traffic, weather, demographics) may be gathered from external sources, e.g. of the Internet or from a database.

Matching context and content. One of the challenges of context-aware advertising systems is the matching of ad content to context (e.g. time, location, etc.). In a traditional advertising business, the marketing group decides when, where, and under what 
circumstances to display the content. With context-aware systems designed to make the decisions about content selection automatically, context information would have to be available in real time. Instead, we recommend giving the advertisers the freedom to specify the conditions in which an ad is shown. Then, the current context of a car and its display(s), the car owner's preferences, and the conditions specified by the advertiser could simply be matched to the context data.

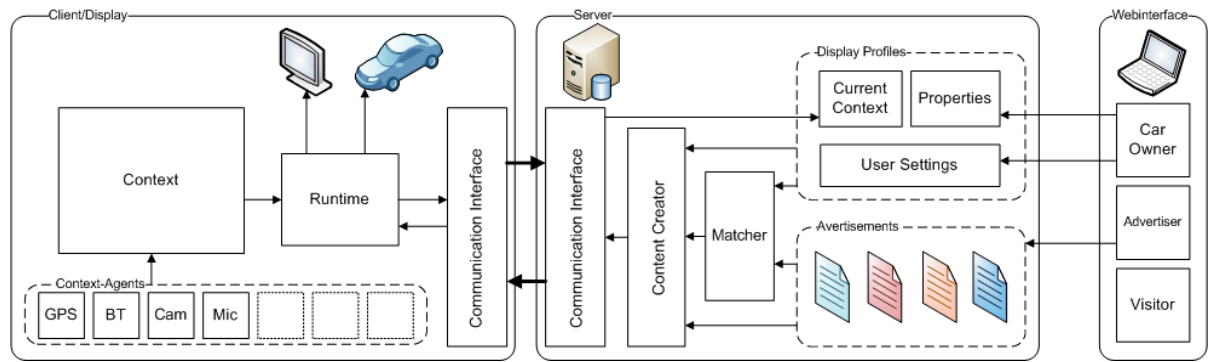

Figure 1: Architecture of the advertising platform. (1) Client side: context agents gather context data (2) Communication Interface: data exchange between client and server side (3) Server side: front-end for advertiser and car owner

\section{A platform for a dynamic contextual advertising system}

As a proof-of-concept, we implemented a prototypical platform that supports contextual advertising on dynamic displays. The system is set up based on a client-server architecture. In order not to rely upon the car's internal computational resources, we implemented a thin client, requiring as few computations and data storage as possible.

The two core functionalities of our car advertising system are 1) the matching of the current context in which the car is situated with the available advertisements, and 2) the smart and efficient exchange of information between multiple cars and the ad server. In the existing prototype, we follow an exact matching approach. Our implementation supports a push-pull communication model: cars send an update of their current context to the server periodically. At the same time, the server itself constantly calculates the best available ad campaign for every client's car. The best-fitting advertisement is then pushed to the client. This is technically realized by dynamically modifying the web page used by the client to display the content.

The front-end of our system consists of two web interfaces - one for the advertiser and one for the car owner. The advertiser's interface provides a way of specifying both the content of the advertising itself, as well as additional settings that define the context in which an ad has to be shown. The decision algorithm needs to know where, when, and under what circumstances to display the ads. The web interfaces were implemented using PHP and currently support the input of locations, date, and time, as well as weather and temperature conditions. The advertiser can select the desired area(s) on a map that is realized by the Google Maps API. The advertiser's interface also supports the upload of pre-designed content (e.g. images). In the car owner's 
interface, the people providing their cars as advertising space can specify their preferences and tell the system what ads they will and will not allow.

The client side includes three different context agents for gathering the context: (1) a GPS agent reporting the location of the client, (2) a time agent providing the time, and (3) a weather agent pulling current weather information and weather forecasts. The server keeps a record of which ads were shown where and on which car. This information can be used to make the car owners context-aware and give them incentives to change their parking behavior (e.g. parking in an highly frequented area will provide a higher revenue than parking in a private backyard), as well as to allow advertisers to assess how successful their campaigns are.

\section{Related Work}

In the following section, we provide an overview of recent work that relates to context-sensitive car advertisements. We focus on three main areas: (1) location-based mobile advertisements supporting the context-based selection of advertisements, (2) suitable technologies for distributing content among mobile devices, and (3) public displays as suitable media for targeting large audiences with advertisements towards.

Examples of mobile applications that support the selection of advertisements based on context are SMMART [7], Ad-me [4] and SmartRotuaari [8]. The first two approaches use Bluetooth, the third one uses WLAN in order to determine the location of a device. All systems support the concept of making decisions about the to-bedisplayed content based on context information.

When it comes to distributing advertisements on mobile clients, two approaches prevail: push (sending information automatically to the client) and pull (sending the information only upon the client's request). Both approaches have been discussed by Ratismor [3] et al. and Varshney [10]. Another interesting approach has been taken by Castro [2]. Castro tries to migrate from a push to a pull model, where users can decide if they wanted to receive more ads after an initial notification.

Finally, research on context-aware advertisements for public displays is closely related to our work, since both types of advertising media try to attract large audiences with different personal backgrounds and interests. Work that focuses on maximizing the exposure of advertisements based on context has been presented by Karam et al. [5] and Kern et al [6]. Both approaches take the interests of the people in the vicinity as context in deciding which advertisements can be tailored to the target group.

\section{Conclusion and Future Work}

Our survey showed that cars have the potential to become an interesting, dynamic and context-aware advertising space in the future. However, it is essential that users stay in control of their vehicles, have an appropriate means for specifying their preferences and are provided with an agreed-upon compensation model. We recommend that location, demographics, time, weather, and history be considered as important contextual parameters. As a proof-of-concept, we implemented a client/server-based plat- 
form that provides a web interface for advertisers and car owners to enter content and preferences. On the client side, several context agents provide real-time data about the current context of the advertising medium. On the server side, a matcher and content creator assemble and prepare web pages for rendering.

Several challenges arise while trying to provide dynamic car advertisements. Although static deployments, such as labels or printed advertisements, are widely available, robust technological solutions for easily setting up context-sensitive advertisements on cars are still under development. National traffic regulations can also lead to additional challenges. For example, according to a telephone interview we conducted with a representative of the German Technical Monitoring Association $(T \ddot{U} V)$, the use of light-emitting or light-reflecting materials is only allowed after passing a strict approval procedure, due to their high risk of distracting other drivers on the road.

As future work, we plan to extend the advertising platform and further develop the contextual displays to make them robust and easy to deploy. We also aim to conduct a larger-scale study to better understand the needs and preferences of advertisers and car owners and to create an appealing business model for both groups.

\section{References}

1. Aalto, L., Göthlin, N., Korhonen, J., and Ojala, T.: Bluetooth and WAP push based locationaware mobile advertising system. In Proceedings of the 2nd international Conference on Mobile Systems, Applications, and Services. MobiSys '04. ACM, New York, NY, 49-58.

2. Castro, J. E. and Shimakawa, H.: Mobile Advertisement System Utilizing User's Contextual Information. In Proceedings of the 7th international Conference on Mobile Data Management, IEEE Computer Society, Washington, DC, 2006.

3. Finin, T., Ratsimor, O., Joshi, A., and Yesha, Y.: eNcentive: A Framework for Intelligent Marketing in Mobile Peer-To-Peer Environments, In: Proceedings of the. Fifth Int'l Conf. Electronic Commerce (ICEC), 2003.

4. Hristova, N., O'Hare, G.M.P.:Ad-me: Wireless Advertising Adapted to the User Location, Device and Emotions. In: Proceedings of the 37th Annual Hawaii International Conference on System Sciences (HICSS'04), 2004.

5. Karam, M., Payne, T. and David, E.: Evaluating BluScreen: Usability for Intelligent Pervasive Displays. In Proceedings of ICPCA 2007.

6. Kern, D., Harding, M., Storz, O., Davis, N., and Schmidt, A.: Shaping how Advertisers See Me: User Views on Implicit and Explicit Profile Capture. In CHI'08 Extended Abstracts. 3363-3368. 2008

7. Kurkovsky, S. and Harihar, K.: Using Ubiquitous Computing in Interactive Mobile Marketing. Personal and Ubiquitous Computing, 10(4):227-240, 2006.

8. Ojala T., Korhonen J., Aittola M., Ollila M., Koivumäki T.,Tähtinen J. \& Karjaluoto H.: SmartRotuaari - Context-aware mobile multimedia services. MUM 2003: 2nd International Conference on Mobile and Ubiquitous Multimedia, 9-18. 2003.

9. Tamminen, S., Oulasvirta, A., Toiskallio, K., and Kankainen, A.: Understanding mobile contexts. Personal Ubiquitous Comput. 8, 2, pages 135-143. 2004.

10. Varshney, U.: Location management for mobile commerce applications in wireless Internet environment. ACM Trans. Interet Technol. 3, pages 236-255, 2003. 